\title{
EVALUATION OF INDIVIDUAL DOSIMETRY IN MIXED NEUTRON AND PHOTON RADIATION FIELDS (EVIDOS). PART II: CONCLUSIONS AND RECOMMENDATIONS
}

\author{
H. Schuhmacher, ${ }^{1,}$, D. Bartlett ${ }^{2}$, T. Bolognese-Milsztajn ${ }^{3}$, M. Boschung ${ }^{4}$, M. Coeck ${ }^{5}$, G. Curzio $^{6}$, \\ F. d'Errico ${ }^{6}$, A. Fiechtner ${ }^{4}$, J.-E. Kyllönen', V. Lacoste ${ }^{3}$, L. Lindborg 7 , M. Luszik-Bhadra ${ }^{1}$, \\ M. Reginatto ${ }^{1}$, R. Tanner $^{2}$ and F. Vanhavere ${ }^{5}$ \\ ${ }^{1}$ Physikalisch-Technische Bundesanstalt, D-38116 Braunschweig, Germany \\ ${ }^{2}$ Health Protection Agency, Chilton, Didcot OX11 0RQ, UK \\ ${ }^{3}$ Institut de Radioprotection et de Sûreté Nucléaire, F-92265 Fontenay-aux-Roses, France \\ ${ }^{4}$ Paul Scherrer Institut, CH-5232 Villigen, Switzerland \\ ${ }^{5}$ Studiecentrum voor Kernenergie- Centre d'étude de l'énergie nucléaire, B-2400 Mol, Belgium \\ ${ }^{6}$ Dipartimento di Ingegneria Meccanica, Nucleare e della Produzione, I-56126 Pisa, Italy \\ ${ }^{7}$ Swedish Radiation Protection Authority, SE-171-16 Stockholm, Sweden
}

\begin{abstract}
The paper presents the main conclusions and recommendations derived from the EVIDOS project, which is supported by the European Commission within the 5th Framework Programme. EVIDOS aims at evaluating state of the art neutron dosimetry techniques in representative workplaces of the nuclear industry with complex mixed neutron-photon radiation fields. This analysis complements a series of individual papers which present detailed results and it summarises the main findings from a practical point of view. Conclusions and recommendations are given concerning characterisation of radiation fields, methods to derive radiation protection quantities and dosemeter results.
\end{abstract}

\section{INTRODUCTION}

The EVIDOS project, supported by the European Commission within the 5th Framework Programme, aims at evaluating state of the art dosimetry techniques in representative workplaces of the nuclear industry with mixed neutron-photon radiation ${ }^{(1)}$. Seven European institutes with recognised expertise in radiation protection instruments and methods joined efforts with end users at nuclear power plants, at fuel processing and reprocessing plants, and at transport and storage facilities. A major task of the project was to develop methods to characterise the neutron component of mixed radiation fields at workplaces and to derive reference values of radiation protection quantities from energy and direction distributions of the neutron fluence.

The results from the project available to date include spectra and dosimetric data for 14 different workplace fields (boiling water reactor, pressurised water reactor, research reactor, fuel processing, storage of spent fuel), instruments and procedures to derive reference values for personal dose equivalent and other radiation protection quantities, and results on the dosimetric and technical performance of personal dosemeters for mixed radiation.

While other papers in this volume describe in more detail the methods developed and the instruments used, this presentation summarises the main results, draws conclusions and discusses general

*Corresponding author: helmut.schuhmacher@ptb.de recommendations. A number of questions, of relevance for the routine monitoring of such complex mixed radiation fields, are addressed in the following sections, e.g., which methods permit the determination of $H^{*}(10)$ and $H_{\mathrm{p}}(10)$; what is the influence of the energy and direction distributions of neutrons on the ratios between $H^{*}(10), H_{\mathrm{p}}(10)$ and $E$; how much do the readings of area monitors and personal dosemeters deviate from these radiation protection quantities and do they give conservative estimates of $E$; do new active (electronic) personal dosemeters give better estimates of $H_{\mathrm{p}}(10)$ than passive ones; and can the overall response of area monitors and personal dosimeters in workplaces of nuclear industry be improved by a suitable calibration?

\section{SUMMARY OF RESULTS}

New methods were developed for the characterisation of workplaces using direction spectrometry ${ }^{(2-4)}$. These new instruments were used together with wellestablished spectrometers, which determine the angleintegrated energy distribution ${ }^{(5)}$. The result is a full characterisation of the radiation fields in terms of the neutron fluence (or fluence rate) as a function of energy and direction $^{(6)}$. Conversion coefficients ${ }^{(7,8)}$ were then used to derive the relevant radiation protection quantities, ambient dose equivalent, $H^{*}(10)$, personal dose equivalent in a slab phantom as used for calibration purposes, $H_{\mathrm{p}}(10)$, and effective dose, $E^{(5,6)}$. 
Results were obtained from a large number of area monitors ${ }^{(9)}$ and personal dosemeters ${ }^{(10)}$. These include commercial devices and instruments developed by the partners outside or within the project.

The first investigations were performed in the simulated workplace fields SIGMA and CANEL at Cadarache (FR) in order to test the various instruments used ${ }^{(11)}$. Then three measurement campaigns were performed in nuclear facilities at Krümmel (DE), Mol (BE) and Ringhals (SE). The result is a comprehensive set of data from 14 workplaces in the nuclear industry, namely at reactors (BWR, PWR, research reactor), at transport casks and for fuel processing. A final campaign is planned in a fuel processing facility.

\section{CONCLUSIONS AND RECOMMENDATIONS}

\section{Characterisation of radiation fields}

The measurements with reference spectrometry revealed significant differences in the energy distributions at the workplaces ${ }^{(5)}$. While all distributions exhibit similar structures - a thermal peak, a rather flat intermediate-energy region and a high-energy peak with a maximum between $100 \mathrm{keV}$ and $1 \mathrm{MeV}$ - the contributions of these neutrons to the fluence varies significantly ${ }^{(5)}$.

The importance of the different neutron energies for dosimetry depends on the fluence-to-dose equivalent conversion coefficient. Because of its strong energy dependence, the contributions of high-energy neutrons to dose equivalent is dominant in all spectra. To give an example, the spectrum with the largest contribution from the thermal and intermediateenergy region was found in a workplace at a research reactor: about $90 \%$ of the neutron fluence is observed in the energy region below $10 \mathrm{keV}$ but these neutrons contribute only about $25 \%$ to ambient dose equivalent.

For the analysis of dosemeter results in the next sections, the average fluence-to-ambient dose equivalent conversion coefficient, $h^{*}$, was used as a parameter to indicate the 'hardness' of a spectrum. Although, in principle, different spectra can lead to the same $h^{*}$, the use of a single parameter allows to categorise spectra for analysing the instruments' behaviour in the different radiation fields. Figure 1 shows $h^{*}$ for the different radiation fields. All spectra at the reactors are soft because they are influenced by massive shielding between the reactor cores and the measurement positions. At the transport casks two types of spectra were observed: Hard spectra at one cask and much softer spectra at another one, which include an additional neutron shield. For fuel processing, the spectra vary significantly depending on the specific shielding conditions. The values of $h^{*}$ indicate that the simulated workplace fields are

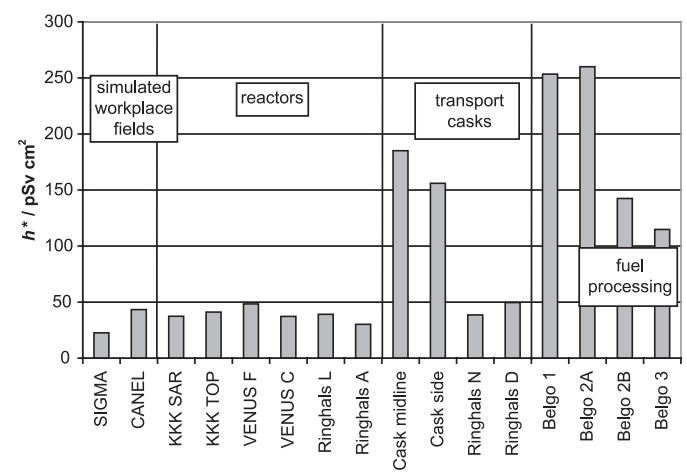

Figure 1. Average fluence-to-ambient dose equivalent conversion coefficient, $h^{*}$, for the different radiation fields investigated.

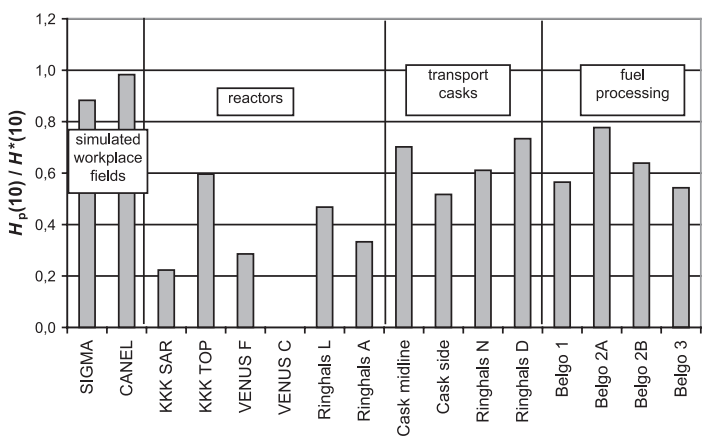

Figure 2. Ratio of personal dose equivalent for the assigned front direction, $H_{\mathrm{p}}(10)$, to ambient dose equivalent, $H^{*}(10)$, for the different radiation fields investigated. For position VENUS C, $H_{\mathrm{p}}(10)$-data are not available because measurements with the direction spectrometer could not be performed.

representative for fields with significant shielding (e.g., at reactors) but not for those with little shielding.

The ratio $H_{\mathrm{p}}(10) / H^{*}(10)$, shown in Figure 2, can be used as a crude indicator of the direction distribution of the neutrons. If neutrons impinge mainly from the normal direction to the front surface of the phantom, like in simulated workplace fields, this ration is close to 1 . The larger the contribution from neutrons from other directions of incidence, the smaller this ratio gets, since neutrons coming from these other directions of incidence are essentially shielded by the phantom. The lowest value of 0.22 was observed for the control rod room underneath the core at the Krümmel power plant where a large isotropic component was present with the main direction being from the top. In general, the direction distributions in the investigated workplaces are more isotropic at reactors and more directed at transport casks and fuel processing. The specific 
results for a particular workplace are influenced by the details of its geometry, in particular with respect to shielding and scattering material.

The photon dose equivalent rate was measured with a FHT $191 \mathrm{~N}$ ionisation chamber and lowpressure proportional counters ${ }^{(10,12)}$. The fractions of photon to total ambient dose equivalent ranged from 17 to $98 \%$ in the workplace fields investigated.

\section{Radiation protection quantities}

The reference method to determine $H^{*}(10)$ of neutrons in unknown radiation fields is that of the Bonner sphere (BS) spectrometry. BS spectrometry is well-established and has been benchmarked against other methods, e.g., calculations and allows $H^{*}(10)$ to be determined with small uncertainties in the order of $5 \%$ (one relative standard uncertainty) if the response matrix of the spectrometer is precisely known and if the data analysis is performed carefully ${ }^{(5)}$.

At present, a reference method to determine $H_{\mathrm{p}}(10)$ does not yet exist. Energy and direction spectrometers are still research tools for which further validation is needed. The results from these instruments can be improved if the angle-integrated spectra from $\mathrm{BS}$ are used as pre-information. In this case the uncertainties are in the order of $30 \%{ }^{(6,10)}$. An alternative method, which may result in similar uncertainties, is based on a superheated drop detector located at $10 \mathrm{~mm}$ depth inside a slab phantom ${ }^{(8)}$. However, further research is needed for this device, e.g., a full characterisation with calibration measurements and transport calculations.

\section{Dosemeter results}

In this paper, the observed responses (readings divided by the reference value or best estimate derived from spectrometry) are analysed in a general way as a function of $h^{*}$. For specific results concerning particular instruments or workplaces the reader is referred to the papers which give the full results from area monitors ${ }^{(9)}$ and personal dosemeters ${ }^{(10)}$.

The area monitors slightly underrespond in hard spectra while the results are between 0.5 and 1.5 (Figure 3). Despite the small underestimates in terms of $H^{*}(10)$, the instruments do provide generally conservative estimates of $H_{\mathrm{p}}(10)$ or $E$. In highly scattered fields the overestimates can be substantial.

For the personal dosemeters, a significant spread of the results is observed (Figure 4). While the best results are obtained in hard spectra, many dosemeters overrespond significantly in soft spectra. The new active (electronic) personal dosemeters (APDs) do not generally give better results than passive ones in terms of the spread of responses - at least for the workplace fields investigated. APDs show, however, a much lower detection limit. In reactor fields, $20 \mu \mathrm{Sv}$

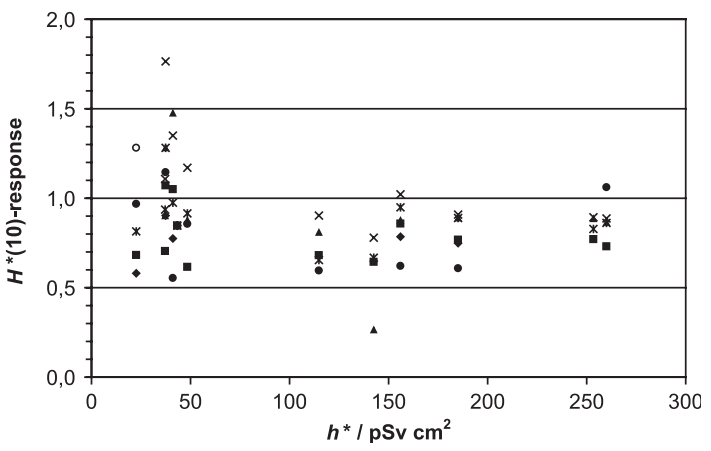

Figure 3. Ambient dose equivalent response of the different area monitors as a function of the average conversion coefficient, $h^{*}$.

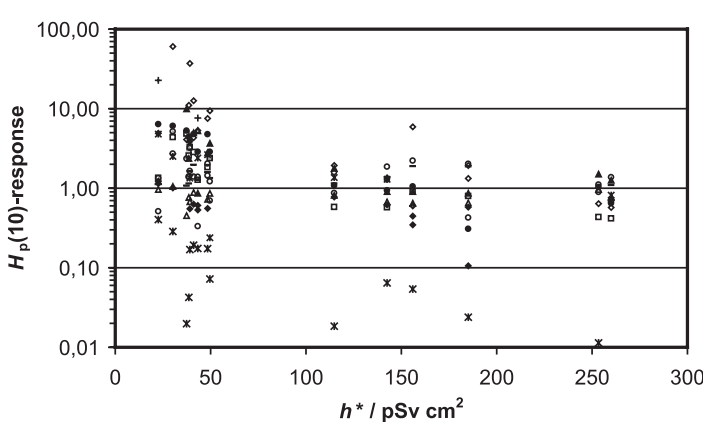

Figure 4. Personal dose equivalent response of the different personal dosemeters as a function of the average conversion coefficient, $h^{*}$.

can be measured with a statistical uncertainty of $10 \%$ with most APDs.

If the application is restricted to highly scattered fields at reactors, the field-specific calibration (e.g., using a simulated workplace field) improves the results for some area monitors and personal dosemeters. Further analysis is needed to understand why for several instruments this procedure does not lead to an improvement.

\section{SUMMARY AND OUTLOOK}

The EVIDOS project will be completed by October 2005. Further measurements in fuel processing are scheduled. To date, a huge number of data have been accumulated in comprehensive measurement campaigns and from accompanying calculations. These include spectra (energy and direction) for 16 radiation fields, about 300 dosimetric data derived from spectrometry, 150 results from area monitors and 500 results from personal dosemeters. Data analysis is partly still in progress, e.g., to check the observed responses with calculations. 


\section{H. SCHUHMACHER ET AL.}

\section{ACKNOWLEDGEMENTS}

This research is partly funded by the European Commission under the auspices of the Euratom 5th Framework Programme: Nuclear Energy, 1998-2002, Contract No: FIKR-CT-2001-00175. The authors wish to express their gratitude to staff at Cadarache, Krümmel, Mol and Ringhals for their help during the campaigns.

\section{REFERENCES}

1. d'Errico, F., Bartlett, D., Bolognese-Milsztajn, T., Boschung, M., Coeck, M., Curzio, G., Fiechtner, A., Kyllönen, J.-E., Lacoste, V., Lindborg, L. et al. Evaluation of individual dosimetry in mixed neutron and photon radiation fields (EVIDOS). Part I: Scope and methods of the project. In press.

2. Luszik-Bhadra, M., Reginatto, M. and Lacoste, V. Measurement of energy and direction distribution of neutron and photon fluences in workplace fields. Radiat. Prot. Dosim. 110, 237-241 (2004).

3. d'Errico, F., Giusti, V., Reginatto, M. and Wiegel, B. A telescope-design directional neutron spectrometer. Radiat. Prot. Dosim. 110, 533-537 (2004).

4. Reginatto, M., Luszik-Bhadra, M. and d'Errico, F. An unfolding method for directional spectrometers. Radiat. Prot. Dosim. 110, 539-543 (2004).

5. Lacoste, V., Assélineau, B., Muller, H. and Reginatto, M. Bonner sphere neutron spectrometry at nuclear workplaces in the framework of the EVIDOS project. In press.
6. Luszik-Bhadra, M., Bolognese-Milsztajn, T., Boschung, M., Coeck, M., Curzio, G., d'Errico, F., Fiechtner, A., Lacoste, V., Lindborg, L., Reginatto, M. et al. Direction distributions of neutrons and reference values of personal dose equivalent in workplace fields. In press.

7. International Commission on Radiation Units and Measurements. Conversion coefficients for use in radiological protection against external radiation. ICRU Report 57 (Bethesda, MD: ICRU) (1998).

8. d'Errico, F., Giusti, V. and Siebert, B. R. L. A new neutron monitor and extended conversion coefficients for $H_{p}(10)$. In press.

9. Tanner, R. J., Bolognese-Milsztajn, T., Boschung, M., Coeck, M., Curzio, G., d'Errico, F., Fiechtner, A., Hager, L. G., Hussien, M., Kyllönen, J-E. et al. Neutron area survey instruments in the EVIDOS project: response in workplaces that have been characterized in terms of energy and direction distributions. In press.

10. Luszik-Bhadra, M., Bolognese-Milsztajn, T., Boschung, M., Coeck, M., Curzio, G., Derdau, D., d'Errico, F., Fiechtner, A., Kyllönen, J.-E., Lacoste, V. et al. Summary of personal neutron dosemeter results obtained within the EVIDOS project. In press.

11. Bolognese, T., Boschung, M., Coeck, M., Curzio, G., d'Errico, F., Lacoste, V., Lindborg, L., Luszik-Bhadra, M., Pochat, J.-L., Reginatto, M. et al. Individual monitoring in workplaces with mixed neutron/ photon radiation. Radiat. Prot. Dosim. 110, 753-758 (2004).

12. Kyllönen, J. and Lindborg, L. Photon and neutron dose discrimination using low pressure proportional counters with graphite and A150 walls. In press. 